\title{
Vehicle-to-Vehicle Visible Light Phase-Shift Rangefinder Based on the Automotive Lighting
}

\author{
Bastien Béchadergue, Luc Chassagne, Member, IEEE, and Hongyu Guan,
}

\begin{abstract}
Vehicle-to-vehicle (V2V) distance measurement is at the heart of the trajectory control performed by the vehicles forming an automated platoon. Although several technologies like radar or lidar are already used with great benefits in such applications, they suffer from interference issues justifying the need of redundant sensors. In this paper, a proof of concept of a visible light rangefinder based on the headlamps and taillights of two consecutive vehicles is brought for the first time. The following vehicle sends with its headlamps a square wave that is received, reconstructed and sent back by the preceding vehicle with its taillights. This echo is received and reconstructed by the following vehicle, and finally compared with the initial signal in order to extract the phase-shift in which the $\mathrm{V} 2 \mathrm{~V}$ distance is contained. A prototype of this system is built using off-the-shelf components and tested for performance evaluation. It is shown that distance measurement is functional up to $25 \mathrm{~m}$ and that the measurement resolution is around $24 \mathrm{~cm}$ at $10 \mathrm{~m}$, a distance typical for platoons in cruise mode, and with a refresh rate of 506 Hz.
\end{abstract}

Index Terms-Distance measurement, visible light, sensors, platooning, cooperative driving.

\section{INTRODUCTION}

$\mathbf{R}$ OAD mobility is without a doubt vital to any society but also has a massive environmental, human, social and economic impact by inducing greenhouse gas emissions, road accidents, noise pollution or traffic congestion. In the context of an increasing demand for transportation, solutions must be proposed to tackle these issues. To that extent, intelligent transportation systems (ITS) have been developed over the past few decades and should lead eventually to widespread autonomous vehicles. Before reaching this end, however, intermediate steps such as platooning might first appear. In platooning, a leading vehicle (LV) is followed by following vehicles (FV) able to adjust automatically their trajectory. This trajectory control would not be possible without distance sensors allowing the members of the platoon to estimate their relative position [1], [2] and thus maintain a constant vehicle-to-vehicle (V2V) distance which optimal value is around $8 \mathrm{~m} \mathrm{[3].}$

Several range-finding technologies are classically used by the automotive industry, among which the most famous are frequency-modulation continuous wave (FMCW) radars, ultrawide band (UWB) radars and time-of-flight (TOF) lidar [4], [5], [6]. On the one hand, FMCW radars, particularly used in adaptive cruise control (ACC) systems, operate generally

The authors are with the Laboratoire d'Ingénierie des Systèmes de Versailles (LISV), UVSQ, Université Paris-Saclay, 10-12 avenue de l'Europe, 78140, Vélizy, France (email: luc.chassagne@uvsq.fr; hongyu.guan@uvsq.fr).

Bastien Béchadergue is also with the Vedecom Institute, 77 rue des Chantiers, 78000, Versailles, France (email: bastien.bechadergue@vedecom.fr). at $24 \mathrm{GHz}$ or $79 \mathrm{GHz}$ and use the frequency shift appearing in a frequency modulated signal after a return-trip between the radar and the target to estimate the distance [5]. Although very efficient, this solution is rather sensitive to interferences [7] and thus to dense traffic situations such as platooning. On the other hand, UWB radars and lidars measure the TOF of a signal during the return-trip to the target. UWB radars operate at 24 or $79 \mathrm{GHz}$ and experience similar issues as FMCW radars, whereas lidars may also be sensitive to interferences [8], in addition to their relatively high cost [6]. Global positioning systems (GPS) could also be used for such purpose but they usually do not provide enough accuracy and do not work without a direct line-of-sight to the satellites. Consequently, simple redundant range-finding solutions, providing measurement errors in the range of a few centimeters over at least $8 \mathrm{~m}$ with a minimum refresh rate of $50 \mathrm{~Hz}$ [3], may be useful.

In [9], [10], a solution called visible light rangefinder (VLR) is proposed. The FV sends a square light signal with its light-emitting diodes (LED) headlamps that is received, reconstructed and sent back by the LV with its LED taillights. The FV receives and reconstructs this echo and then compares it with the signal originally sent in order to extract a phaseshift proportional to $\mathrm{V} 2 \mathrm{~V}$ distance. The simulation study carried out in [9] shows that this system could ensure distance measurement over $30 \mathrm{~m}$ with a resolution under $30 \mathrm{~cm}$ and at a refresh rate of $267 \mathrm{~Hz}$ so that it would be an interesting complement in platooning configurations. In this paper, a proof of concept of the VLR is brought for the first time, along with its performances evaluation. It is shown that our VLR prototype is able to estimate the longitudinal distances over 25 m with a resolution of around $24 \mathrm{~cm}$ at $10 \mathrm{~m}$, a V2V distance typical for platoons in cruise mode, and with a refresh rate of $506 \mathrm{~Hz}$.

Before reaching these results, the general working principles of the VLR are first reminded in Section II whereas its practical implementation is detailed in Section III. The experiments carried out for performance evaluation are described in Section IV, along with their results. Finally, Section V gives some concluding remarks and relevant future works.

\section{Design of the Visible Light RANGefinder}

\section{A. General Working Principles}

Classical rangefinders such as FMCW radars, UWB radars or lidars generally work according to the same core principle. The system sends a signal that is reflected back by the target [4]. The resulting echo is then detected by the system and 
processed to recover the system/target distance. Since these technologies use radio or light carriers that are coherent, the echo is indeed strong enough to be exploited. Unfortunately, the echo of the light signal produced by non-coherent polychromatic and diffuse sources such as LED headlamps or taillights is generally too weak to provide relevant information. Therefore, our VLR is based on an active reflection principle. The target, here the $\mathrm{LV}$, receives the light signal transmitted by the system, here the FV, and reconstructs it before re-emitting it. This way, the echo received by the FV can be properly detected and processed.

The second core principle of the VLR is the phase-shift measurement on which the distance estimation relies. If the FV sends a periodic signal of fixed frequency, then its echo will have the same frequency but will also contain a phaseshift proportional to the V2V distance. This technique, when combined with active reflection, is called distance estimation via asynchronous phase-shift (DEVAPS) [11], and is illustrated in the case of the VLR by Fig. 1 [9].

The VLR thus operates as follows. First, the FV emits a square signal $s_{e}$ of frequency $f_{e}$ by driving one of its LED headlamps with a dedicated emitter circuit. After free space propagation, this signal is detected by a photodiode (PD) on the LV and turned into an electrical signal $s_{p^{\prime}}$ that is processed by the receiver in order to remove the channel noise and recover a square signal $s_{r^{\prime}}$ of frequency $f_{e}$. A phase-locked loop (PLL) may be added in order to recover a $50 \%$ duty cycle square signal $s_{e^{\prime}}$ of frequency $f_{e}$, which is then emitted by the LV toward the FV with one of its taillights. This light signal is in turn detected by a PD on the FV, after free space propagation. The resulting electrical signal $s_{p}$ is, using the same process as previously, reconstructed in a square signal $s_{r}$ of frequency $f_{e}$. This reconstructed signal has, with the signal first transmitted $s_{e}$, a phase-shift $\varphi$ linked with the V2V distance $d$ and the light velocity $c$ by:

$$
d=\frac{c}{2 f_{e}} \cdot \frac{\varphi}{2 \pi} \text {. }
$$

This equation, when differentiated, shows that the distance measurement resolution depends on the frequency of operation. More precisely, increasing $f_{e}$ enhances the measurement resolution. However, $f_{e}$ also impacts the non-ambiguity range, defined as $c /\left(4 f_{e}\right)$, which is the range over which a measure can be given without any ambiguity. Therefore, the frequency of operation must be chosen carefully. On the other hand, the distance measurement resolution is closely related to the phase-shift measurement resolution, which will depend on the method used for its estimation.

\section{B. Phase-Shift Measurement Technique}

The phase-shift measurement process first starts with an heterodyning step. The signals sent and received $s_{e}$ and $s_{r}$ are transposed to lower frequency signals $s_{e h}$ and $s_{r h}$ using a heterodyning block, implemented with a D flip-flop gate cadenced by a clock $s_{h}$. This clock has a frequency $f_{h}$ linked with the frequency of operation $f_{e}$ by a heterodyning factor $r$ such that:

$$
f_{h}=\frac{r}{r+1} f_{e}
$$

This factor $r$ introduces a small time difference $1 /\left(r f_{e}\right)$ between the periods of the input signal $s_{e}$ or $s_{r}$ and the heterodyning clock $s_{h}$. Therefore, although the values output by the heterodyning block are separated by $1 / f_{h}$ seconds in reality, they capture the evolution of the input signal with a time resolution of $1 /\left(r f_{e}\right)$ seconds. In other words, our heterodyning block acts as a sampler of rate $1 /\left(r f_{e}\right)$ that requires $r+1$ input periods of input signal to reconstruct one full period of output signal. Therefore, the heterodyned signals $s_{e}$ and $s_{r}$ have an intermediate frequency $f_{i}$ given by:

$$
f_{i}=\frac{f_{e}}{r+1} .
$$

This behavior is illustrated by Fig. 2, when $r=10$. Once the heterodyned versions $s_{e h}$ and $s_{r h}$ of $s_{e}$ and $s_{r}$ are obtained, they are combined through a simple XOR gate in order to get a phase-shift signal $s_{\varphi}$ containing phase-shift pulses of rate $2 f_{e} /(r+1)$ and of certain widths. Note, however, that this heterodyning step might introduce errors in the phase-shift pulse widths. As shown on Fig. 2 with the fifth rising edge of $s_{r}, s_{h}$ might not detect the transition of the input at the exact time of occurrence, which will modify the pulse width of the heterodyned signal - here $s_{r h}$ which should follow the black dotted line - and thus the phase-shift signal. In practice, this error is, in terms of distance, of at most $c /\left(2 r f_{e}\right)$.

Once the phase-shift pulses are produced, their width is estimated using the auto-digital phase-measurement technique [12], [13] described on Fig. 3(a) and illustrated by Fig. 3(b). First, the phase-shift pulses $s_{\varphi}$ are isolated by groups of $N$ $(N=4$ on Fig. 3$)$ and then combined with a clock signal $s_{\text {clock }}$ of high-frequency $f_{\text {clock }}$ in order to get a signal $s_{\varphi^{\prime \prime}}$ composed of several sub-pulses fitting the original phase-shift pulses. The number of sub-pulses $M$ corresponding to $N$ phase-shift pulses is finally counted so that the overall duration $T_{m}$ of these pulses can be estimated using:

$$
T_{m}=\frac{M}{f_{\text {clock }}}
$$

which corresponds to a mean phase-shift estimate $\varphi_{m}$ :

$$
\varphi_{m}=2 \pi \cdot \frac{T_{m}}{N T_{i}}
$$

with $T_{i}=1 / f_{i}$ the period of the heterodyned signal. By combining (5) with (3) and (4), we can get a more explicit form of $\varphi_{m}$ :

$$
\varphi_{m}=\frac{2 \pi M f_{e}}{(r+1) N f_{\text {clock }}}
$$

that can then be converted into a distance estimate $d_{m}$ by mixing it with (1):

$$
d_{m}=\frac{c}{2} \cdot \frac{M}{(r+1) N f_{\text {clock }}}=\frac{c}{2} \cdot \frac{M f_{i}}{N f_{e} f_{\text {clock }}} .
$$

The refresh rate of the measurement $f_{\mathrm{VLR}}$ is then given by:

$$
f_{\mathrm{VLR}}=\frac{2 f_{e}}{(r+1) N} .
$$




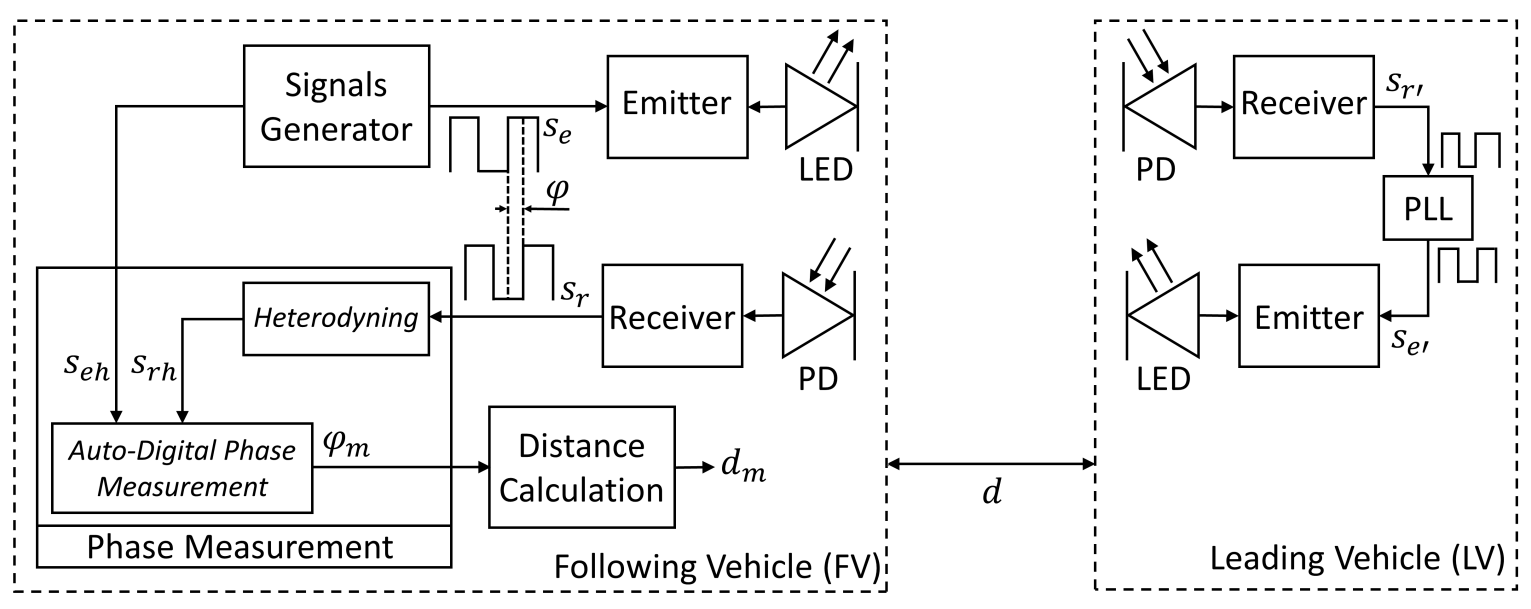

Fig. 1. Block diagram of the visible light phase-shift rangefinder system.

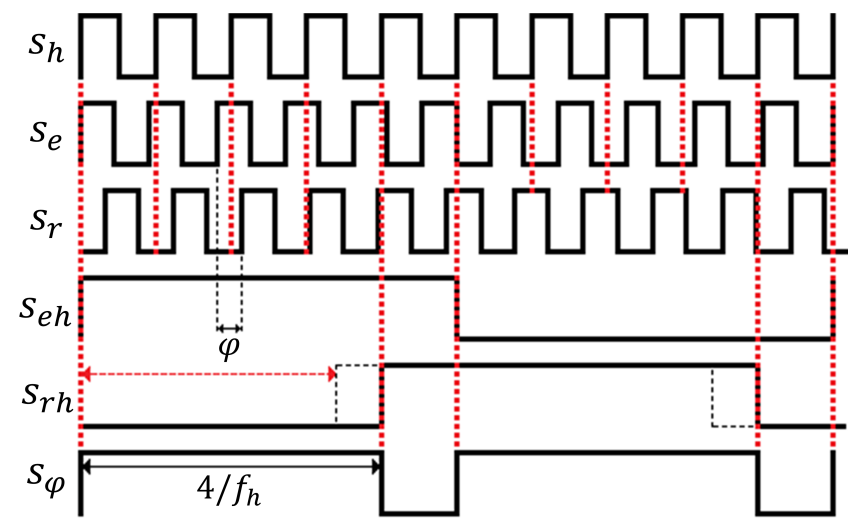

Fig. 2. Illustration of the heterodyning process with $r=10$ on a signal $s_{e}$ synchronized with the heterodyning clock $s_{h}$ and on a signal $s_{r}$ not synchronized and having a phase-shift $\varphi$ with $s_{e}$. The resulting heterodyned signals $s_{e h}$ and $s_{r h}$ are then used to produce the phase-shift signal $s_{\varphi}$ through a XOR gate.

\section{Theoretical Error Analysis}

The distance measurement error $\delta d_{m}$ can be obtained by differentiating (7) in its second form:

$$
\delta d_{m}=d_{m}\left[\frac{\delta M}{M}+\frac{\delta f_{i}}{f_{i}}-\frac{\delta N}{N}-\frac{\delta f_{e}}{f_{e}}-\frac{\delta f_{\text {clock }}}{f_{\text {clock }}}\right] .
$$

As already explained in [9], $\delta f_{\text {clock }}$ and $\delta f_{e}$ represent the frequency drifts of the signals $s_{\text {clock }}$ and $s_{e}$ that may be caused by temperature variations or age. $\delta N$ accounts for the potential non-synchronization between the gate signal $s_{\text {gate }}$ and the phase-shift signal $s_{\varphi}$ whereas $\delta M$ stands for the \pm 1 counting error that may be contained in $M$.

$\delta f_{i}$ gathers, on its side, three distinct sources of errors. The intermediate frequency $f_{i}$ is indeed obtained from the combination between the heterodyning clock $s_{h}$ and the received clock $s_{r}$. On the one hand, $s_{h}$ is generated from the master clock $s_{\text {clock }}$ by a PLL and may thus experience frequency drifts that will impact $f_{i}$. On the other hand, $s_{r}$ might have in reality a frequency slightly varying around $f_{e}$ and not perfectly equal to this value because it is obtained after transmission, reception and processing - especially filtering

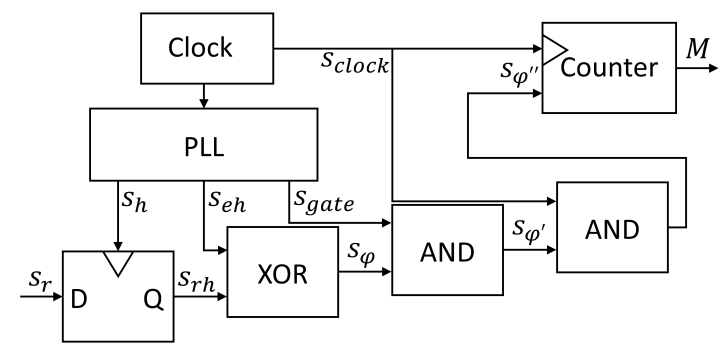

(a)

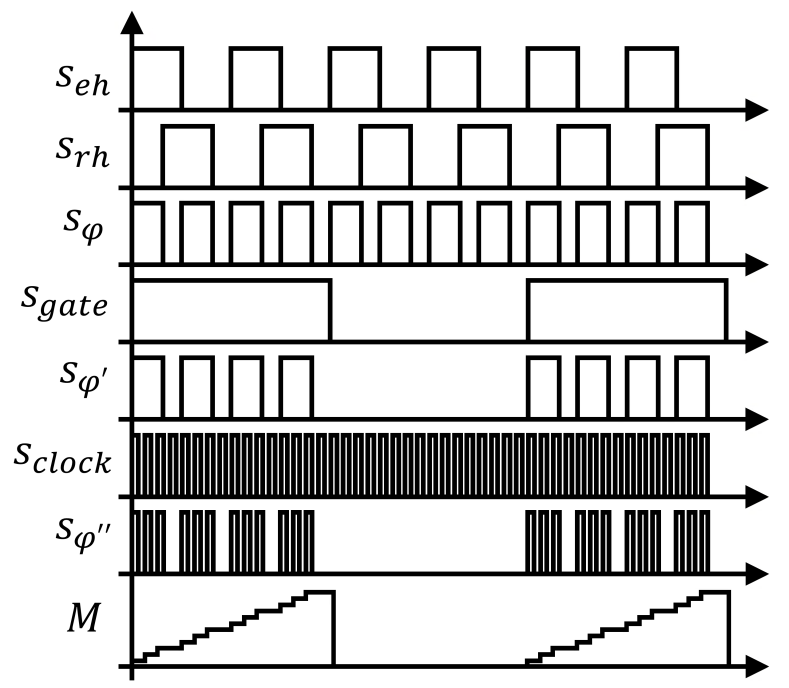

(b)

Fig. 3. (a) Block diagram of the phase-shift measurement system and (b) illustration of its working principles.

and comparison - with components of limited bandwidth, which will undoubtedly introduce some distortions. Slight variations in the phase of $s_{r}$ will then result in phase variations of $s_{r h}$ and thus of $s_{\varphi}$, which induces eventually errors in the clock count $M$. Fig. 4, obtained from (7) with $f_{e}=1 \mathrm{MHz}$, $r=3999, N=1$ and $f_{\text {clock }}=100 \mathrm{MHz}$, shows for example that an additional phase-shift in $s_{\varphi}$ of $1^{\circ}$ results in 1111 additional clock ticks in $M$, which leads to a measurement error of around $42 \mathrm{~cm}$. 


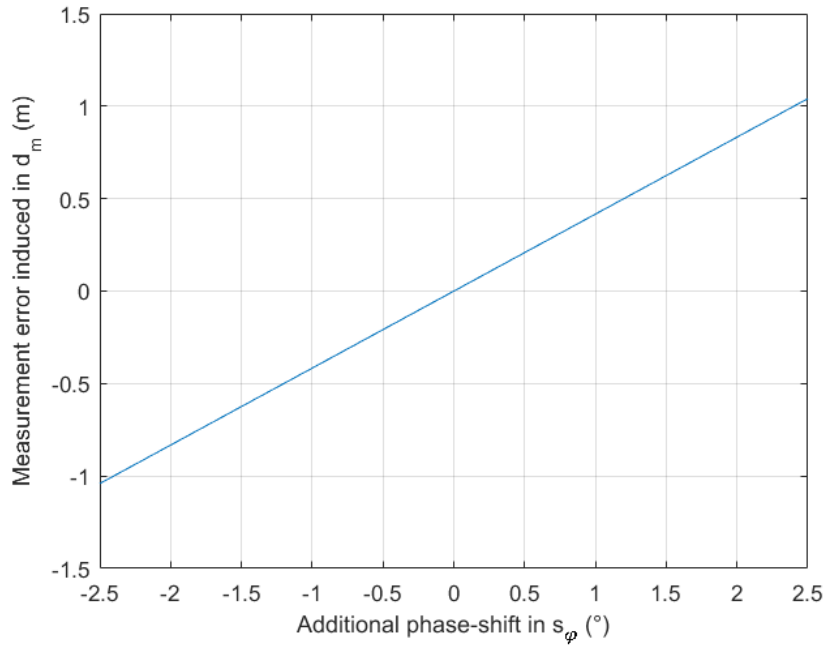

Fig. 4. Evolution of the distance measurement error induced by an additional phase-shift in $s_{\varphi}$, with $f_{e}=1 \mathrm{MHz}, r=3999, N=1$ and $f_{c} l o c k=100$ $\mathrm{MHz}$.

Finally, $\delta f_{i}$ hides a third source of errors, which is the heterodyning-induced error of at most $c /\left(2 r f_{e}\right)$ already detailed in the Section II.B, and illustrated by Fig. 2 .

Note, however, that this error analysis is carried out in a static environment, whereas in real platooning configurations, the $\mathrm{V} 2 \mathrm{~V}$ distance is always varying. These distance variations may impact the distance measurement resolution through the well-known Doppler effect. For example, if the FV is approaching the LV from an initial position $x_{0}$ at a constant speed $v_{0}$, then if a rising edge of $s_{e}$ is sent at time $t_{e}$, while the $\mathrm{V} 2 \mathrm{~V}$ distance is $d\left(t_{e}\right)$, its echo will not be received $2 d\left(t_{e}\right) / c$ seconds later, as in the static case, but with a delay slightly smaller. If $t_{r}$ is the time of reception and $\tau=t_{r}-t_{e}$ the delay of reception, it can be shown that [16]:

$$
\tau=t_{r}-t_{e}=-\frac{x_{0}+v_{0} t_{e}}{c}-\frac{x_{0}+v_{0}\left(t_{e}-\frac{x_{0}+v_{0} t_{e}}{c}\right)}{c+v_{0}} .
$$

During this delay, the FV travels a distance $v_{0} \tau$ which could interact with the distance measurement process, especially the heterodyning step, and thus generate a measurement error $d_{m, p e r f}$. It can be shown that the mathematical form of this error is [16]:

$$
\delta d_{m, p e r f}=\frac{v_{0} \tau_{n}}{2}+\frac{v_{0} \alpha_{n}}{r f_{e}}+\frac{c \alpha_{n}}{2 r f_{e}},
$$

where $\alpha_{n} \in\left[0,1\left[\right.\right.$ is a normalization parameter and $t_{n}$ is the propagation delay of the specific rising edge of $s_{r}$ triggering a rising edge of $s_{r h}$ and thus the end of the phase-shift pulse. We can observe that the first two terms depend on the vehicle speed $v_{0}$ whereas the third one is independent of this parameter. Therefore, we can deduce that the first two terms are dynamic errors, only appearing when there is a relative movement between the VLR and the target, whereas the last term is a static error that is always present.

Given the speed of light $c$, the delay $t_{n}$ usually does not exceed $0.5 \mu \mathrm{s}$, so that the first term in (11) is lower than 1.5 $\mu \mathrm{m}$ when the speed of approach $v_{0}$ is $20 \mathrm{~km} / \mathrm{h}$. At the same time, the second term in (11) is no larger than $1.5 \mathrm{~nm}$ when $r$
$=3999$ and $f_{e}=1 \mathrm{MHz}$. We can thus conclude that relative movements between the two vehicles only induce negligible additional error. On the other hand, we recognize from the third term in (11) the heterodyning error already contained in $\delta f_{i}$. This error can reach at most $3.75 \mathrm{~cm}$ with $r=3999$ and $f_{e}=1 \mathrm{MHz}$, which means the static heterodyning error remains the main source of errors even in dynamic conditions.

\section{PROTOTYPE IMPLEMENTATION}

\section{A. Signal Emission: LED and LED Driver}

The signal emission is made by the VLR prototype using an LED light source controlled by an LED driver. These components correspond to the blocks 'LED' and 'Emitter' in Fig. 1. The light source used on the FV side is a commercial off-the-shelf (COTS) white headlamp based on a Nichia NC4W121A high-power phosphor LED. The overall module is controlled with a forward current limited to $600 \mathrm{~mA}$, which gives a maximum luminous intensity of $16750 \mathrm{~cd}$ compliant with standard regulations for low beam headlamps [14].

On the LV side, another COTS white headlamp is used, but is this time underdriven to meet taillights regulations [15]. Both light sources have a $3 \mathrm{~dB}$ bandwidth of $1.4 \mathrm{MHz}$, so the frequency of operation $f_{e}$ is limited to $1 \mathrm{MHz}$. In this case, the non-ambiguity range is $75 \mathrm{~m}$, which is larger than the range we want the VLR to cover. In order to drive these lamps, a specific LED driver is designed and built. This driver converts the $1 \mathrm{MHz}$ small current complementary metal oxide semiconductor (CMOS) square signal of command $s_{e}$ or $s_{e^{\prime}}$, produced by a field programmable gate array (FPGA), into the $600 \mathrm{~mA}$ control signal. It is thus simply a metal oxide semiconductor field effect transistor (MOSFET), which gate is controlled by the signal of command, first amplified with an optocoupler so that the appropriate amount of current can flow through the LED.

\section{B. Signal Reception: PD and Processing Card}

After their emission, the light signals are collected and processed by dedicated electronic cards in order to be reconstructed: this is the signal reception chain, corresponding to the blocks 'PD' and 'Receiver' in Fig. 1. These receiving chains, identical on both ends of the system, are composed of the different stages represented on Fig. 5(a). First, the light signal is collected by a PD which produces a photocurrent turned into a voltage and amplified by a transimpedance amplifier (TIA). This signal is then bandpass filtered to remove as much noise as possible and isolate the $1 \mathrm{MHz}$ component, which is, after amplification, sent to a comparator for zero-crossing detection. The resulting CMOS square signal, of frequency $f_{e}$, is then used to feed the LED driver in the case of the LV or the FPGA for phase-shift measurement in the case of the FV.

In practice, these different stages are implemented with, firstly, a Thorlabs PDA8A, used as PD and TIA of bandwidth $50 \mathrm{MHz}$ and gain $50 \mathrm{kV} / \mathrm{A}$, that is then connected to a custommade reconstruction card. This card, shown on Fig. 5(b), embeds an 8-th order bandpass filter of center frequency 1 $\mathrm{MHz}$ and bandwidth $100 \mathrm{kHz}$, amplification stages providing a maximum gain of $200 \mathrm{~V} / \mathrm{A}$ and a high-speed comparator 
(a)

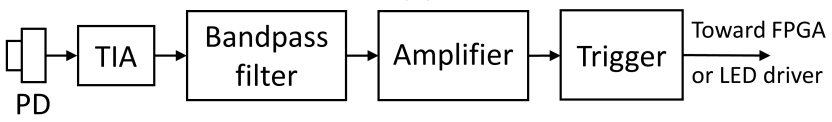

(b)

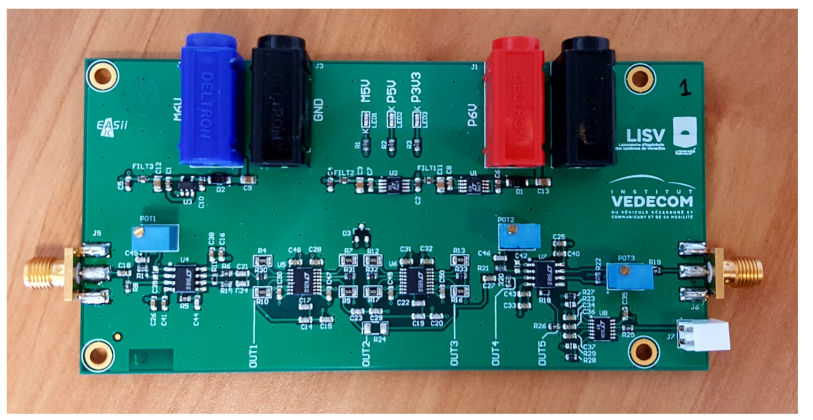

Fig. 5. (a) Processing chain of the reception and reconstruction cards and (b) view of the reconstruction card.

so that it can turn noisy input signals of a few millivolts into a clean square CMOS signal. Fig. 6 illustrates this processing chain with traces observed with a Keysight MSO$\mathrm{X}$ 3054A oscilloscope. The blue signal is the input square signal, generated with a waveform generator Agilent 33500B, of amplitude $20 \mathrm{mV}_{p p}$ and frequency $1 \mathrm{MHz}$, over which is superimposed a $10 \mathrm{MHz}$ Gaussian noise of amplitude 250 $\mathrm{mV}_{p p}$. The resulting input $\mathrm{SNR}$ is measured at $5 \mathrm{~dB}$, as shown by its its fast Fourier transform (FFT) in purple. The black signal is the result obtained after filtering. It has a clear sine wave shape, even though its amplitude is varying. Finally, the red signal is the square wave reconstructed by the high-speed comparator, which has a stable frequency of $1 \mathrm{MHz}$, when measured with a frequency counter Agilent 53132A.

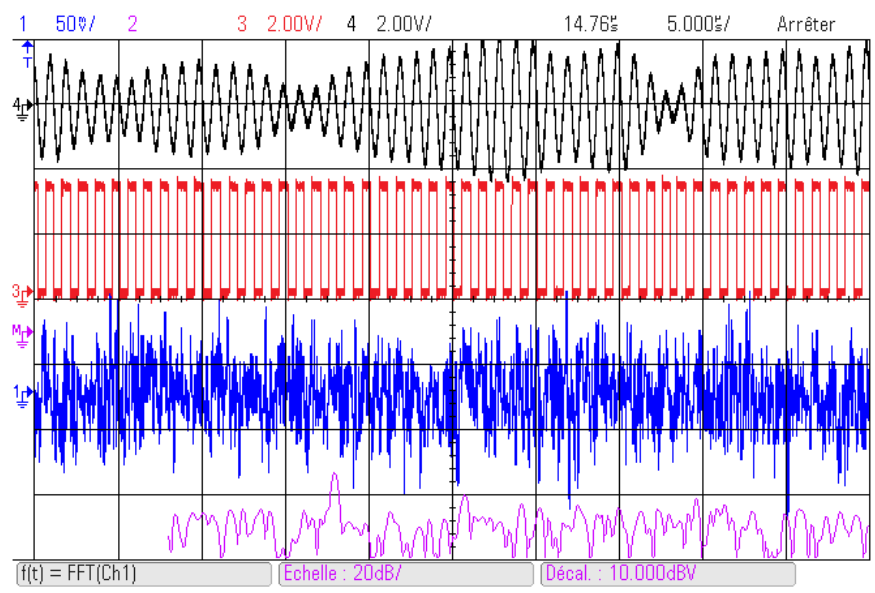

Fig. 6. Illustration of the processing chain of the VLR: in blue, input signal of peak-to-peak amplitude $20 \mathrm{mV}_{p p}$ and $\mathrm{SNR}=5 \mathrm{~dB}$, in purple, its FFT, in black, signal obtained after band-pass filtering and in red, square signal reconstructed by the high-speed comparator.

Although not clearly visible on Fig. 6, this processing chain introduces a delay between the input and output signals. By sending a noiseless sine wave and observing the phase-shift contained in the reconstructed square wave, we can observe that this delay is around 933 ns with the card used on the
LV and 928 ns with the card used on the FV, results that are, in addition, variable with the atmospheric conditions and especially with the temperature. These delays and their variations will necessarily have an impact on the final phaseshift and thus on the distance estimated, as we will see in Section IV.B, after detailing the implementation of the phaseshift measurement algorithm.

\section{Phase-Shift Measurement Algorithm}

Once the signal received from the LV is reconstructed into a stable square signal $s_{r}$, it is compared with the original square wave $s_{e}$ for phase-shift measurement. This phase-shift measurement step is performed digitally by an FPGA which has actually three main roles: generate $s_{e}$, heterodyne $s_{e}$ and $s_{r}$ and, finally, measure their phase-shift, which corresponds to the blocks 'Signals Generator' and 'Phase Measurement' in Fig. 1. Here, the signal $s_{e}$ of frequency $f_{e}=1 \mathrm{MHz}$ is first generated from the internal clock of the FPGA, through a PLL, and continuously fed to the LED driver. The PLL also generates the counter clock $s_{\text {clock }}$ of frequency $f_{\text {clock }}=100$ $\mathrm{MHz}$ used for auto-digital phase measurement. Finally, a second PLL generates the heterodyning clock $s_{h}$ of frequency $f_{h}$.

Regarding this clock, it can be shown through simulations that an heterodyning factor $r=3999$ leads to an interesting compromise between measurement error and refresh rate [10]. Fig. 7, obtained from (3), also shows that it gives a rather large measurement refresh rate. The resulting heterodyning frequency $f_{h}$ is in this case $999750 \mathrm{kHz}$. Unfortunately, the resolution of the internal PLL is not sufficient to support this exact frequency, which is measured instead at $999746.9 \mathrm{kHz}$ with a frequency counter Agilent 53132A, a value corresponding to a heterodyning factor $r=3950.007$. Therefore, the final refresh rate of the measurement is $506 \mathrm{~Hz}$. In practice, this clock is used to cadence a D flip-flop gate coded in hardware description language (HDL), as the rest of the autodigital phase measurement algorithm represented on Fig. 3(a). This algorithm outputs the number of clock ticks $M$ that is stored and then converted into phase-shifts and distances with MATLAB.

Fig. 8 shows the evolution of the phase-shift measured by our FPGA implementation when the true phase-shift varies from 0 to $360^{\circ}$, by steps of $5^{\circ}$. From 0 to $180^{\circ}$, the measure is linear with a unit positive slope, whereas from $180^{\circ}$ to $360^{\circ}$, the slope is -1 . The inflection at $180^{\circ}$ is due to the XOR combination of $s_{e}$ and $s_{r}$ which only exhibits the phase-shift over a half cycle. Note that each point in this figure is actually the average value of 512 consecutive measures. Each measure contains a small error induced by the limited resolution of the heterodyning step evoked previously. However, these errors remain under $3 \mathrm{~cm}$ which is in accordance with the maximum error $c /\left(2 r f_{e}\right)$ equal here to $3.79 \mathrm{~cm}$. This behavior confirms nevertheless that our phase-shift measurement algorithm is fully functional. 


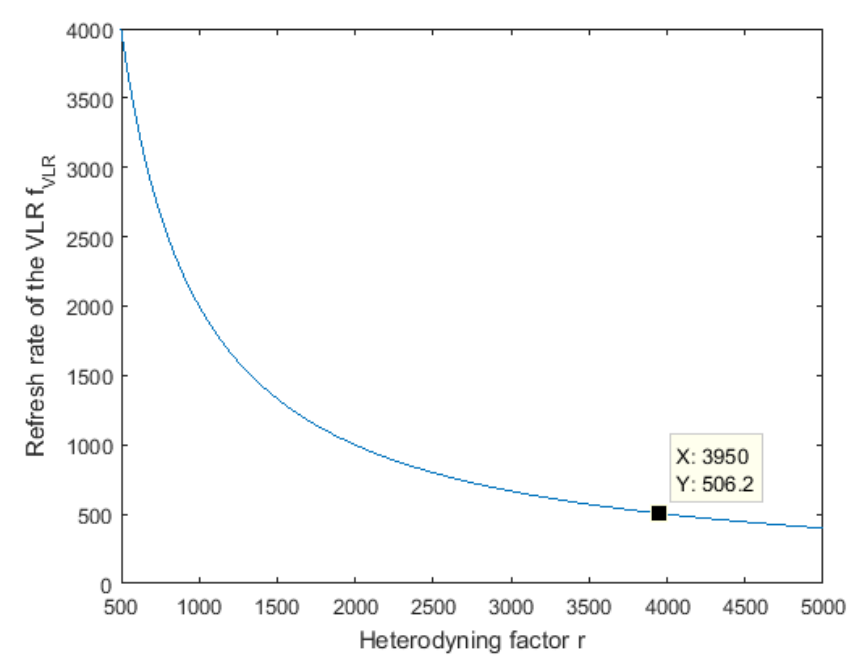

Fig. 7. Evolution of the refresh rate $f_{\mathrm{VLR}}$ with the heterodyning factor $r$ when $f_{e}=1 \mathrm{MHz}$ and $N=1$.

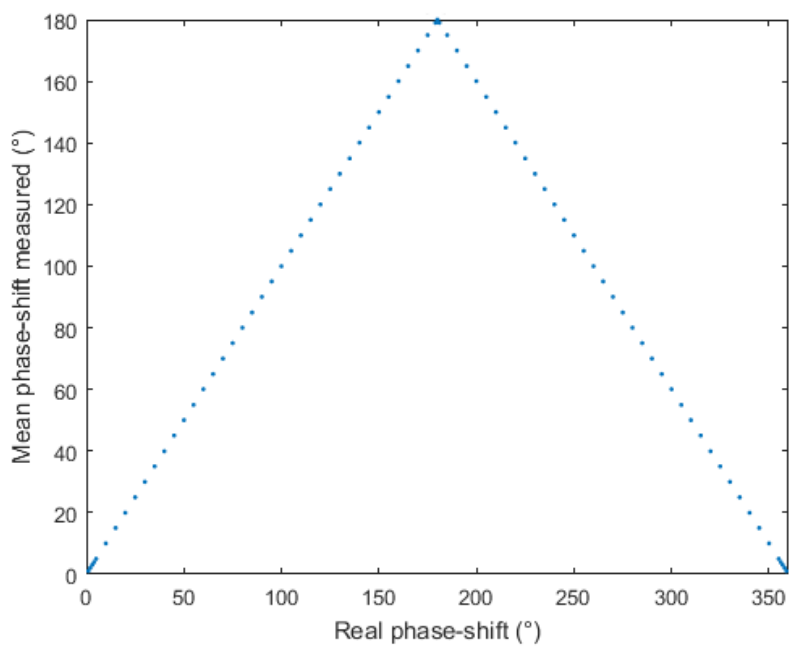

Fig. 8. Evolution of the average phase-shift measured by the dedicated FPGA algorithm with an heterodyning factors $r=3950.007$.

\section{Performances Evaluation of the Prototype}

\section{A. Experimental Set-Up and Protocol}

Fig. 9 is the translation (a) in block diagram and (b) in reality of Fig. 1 with each function replaced by one of the core bricks just detailed.

In practice, the FV and LV are modeled by moving tables on which all the equipment is installed. In addition, the FV end is equipped with a laser rangefinder in order to determine the 'true' distance between both vehicles. This rangefinder, a Laserliner LaserRange-Master 40, can measure distances up to $30 \mathrm{~m}$ with a typical resolution of $\pm 2 \mathrm{~mm}$ and was used in a controlled laboratory environment where no interferences were degrading its performances. Therefore, we can consider the distance obtained is reliable enough and corresponds to the 'true' V2V distance. The knowledge of this value is obviously crucial in order to determine the measurement performances of our VLR.

These range and resolution performances are evaluated using a simple protocol. The table modeling the FV is fixed

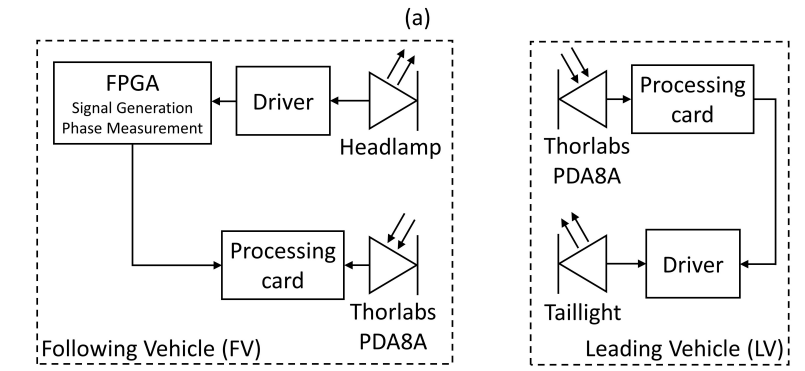

(b)

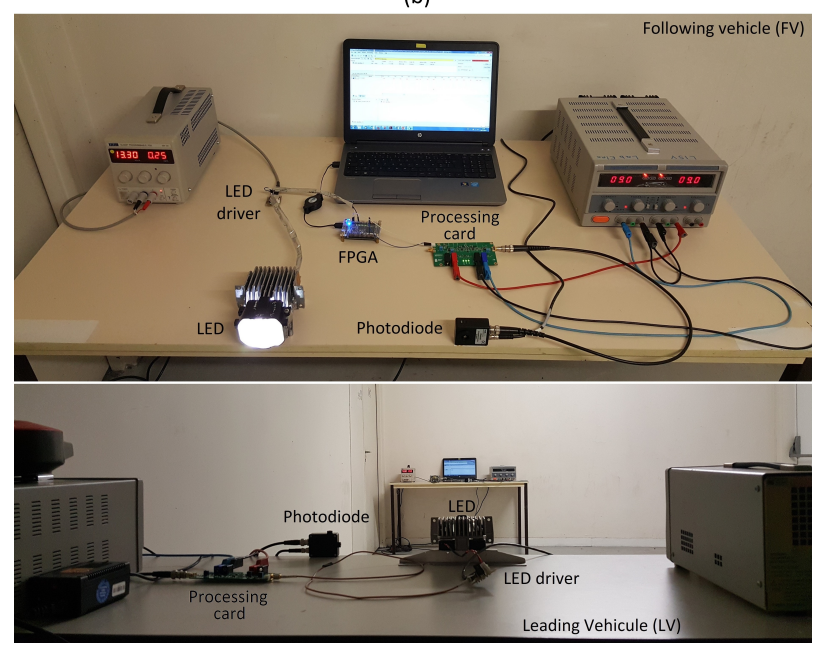

Fig. 9. (a) Structure of the set-up used during the VLR experiments and (b) photo of this set-up with at the top the FV end and at the bottom the LV end.

whereas the table representing the $\mathrm{LV}$ is moved in order to vary the $\mathrm{V} 2 \mathrm{~V}$ distance from $5 \mathrm{~m}$ to $25 \mathrm{~m}$ by steps of $50 \mathrm{~cm}$. Both tables are kept on the same longitudinal axis so that both vehicles can be considered aligned in a straight line. At each step, the 'true' distance is first measured with the laser rangefinder and 4096 consecutive distance estimations are then performed with the VLR. Consequently, the measured are performed in static conditions. Note, however, that the relative movement between the vehicles and the resulting Doppler effect has theoretically a negligible impact on the measurement performances, as explained in Section II.C and demonstrated in [16].

\section{B. General Behavior and Measurement Correction}

Fig. 10 shows the evolution of the average distance measured against the real distance when following the experimental protocol just described.

The most striking result is obviously the inflection point around $10.5 \mathrm{~m}$. From $5 \mathrm{~m}$ to $10.5 \mathrm{~m}$, the distance estimation is indeed decreasing whereas from $11 \mathrm{~m}$ to $25 \mathrm{~m}$, it is rising. This behavior is induced by the processing delays added by the reconstruction cards used on both ends of the VLR. These delays are such that the overall phase-shift between $s_{e}$ and $s_{r}$ at $5 \mathrm{~m}$ is larger than $\pi$. Consequently, as the V2V distance and thus the return trip TOF increases, the overall phase-shift gets larger which leads to a decrease in the value output by the VLR, in accordance with Fig. 8. Then, at $10.5 \mathrm{~m}$, the sum of the various phase-shifts is such that $s_{e}$ and $s_{r}$ are in phase, 


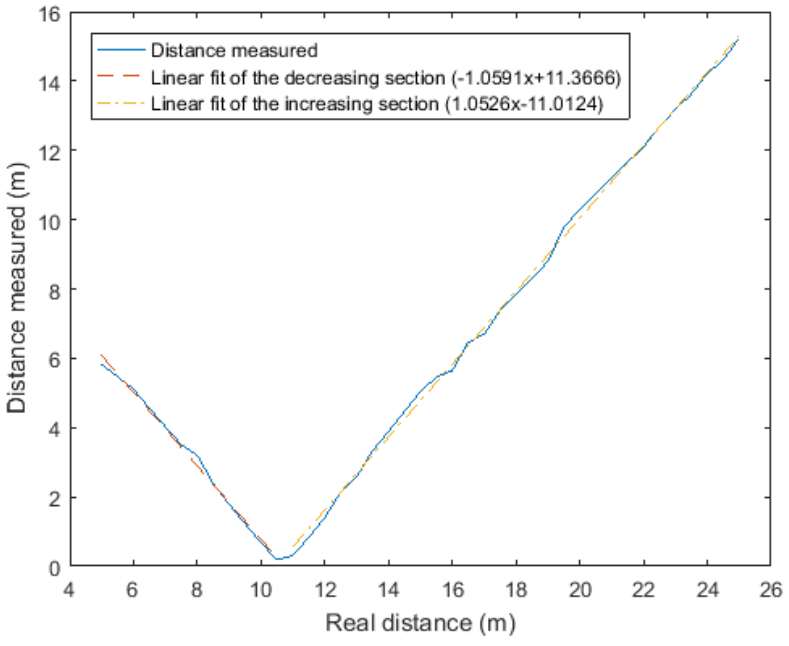

Fig. 10. Evolution of the average distance measured by the VLR against the real distance with the linear fits of its decreasing and increasing sections.

which leads to a distance measured null. After this value, the phase-shift is again positive, but this time below $\pi$, hence the rising section on Fig. 10.

Consequently, the inflection point observed on Fig. 10 is simply the consequence of this general shift caused by the electronic delays already observed in Section III.B. The phase-shift $\varphi_{m}$ eventually measured is indeed the sum of the real $\mathrm{V} 2 \mathrm{~V}$ phase-shift $\varphi$ with an additional phase-shift $\varphi_{\text {elec }}$ corresponding to these electronic delays. Then, with reference to (1), we understand that this undesired phase-shift will introduce a measurement error $\delta d_{\text {elec }}$ of:

$$
\delta d_{\text {elec }}=\frac{c}{2 f_{e}} \cdot \frac{\varphi_{\text {elec }}}{2 \pi} .
$$

Here, $\delta d_{\text {elec }}$ is around $10.5 \mathrm{~m}$. Fortunately, we can observe from the linear fits on Fig. 10 that both the decreasing and rising sections are rather linear, with an absolute slope close to unity, which means the electronic delays remain actually rather constant. In other words, this linear behavior shows that it should be possible to apply a correction rule on these first results to remove the electronic delays and thus the inflection point with the corresponding measurement error.

In practice, the measures are corrected as follows: first, an additional and controlled delay is added so that the final phase-shifts remain between 0 and $\pi$, whatever the true $\mathrm{V} 2 \mathrm{~V}$ distance. Then, a linear fit of the averaged distance measurement is performed in order to define the offset that is finally removed. Note that such a correction assumes the various delays remain constant with time, which is here relevant given the duration of the whole experiment. However, in real conditions, this correction step will have to be repeated periodically because, for example, of the temperature variations the vehicle can experience throughout a journey.

Figure 11 proposes an example of auto-calibration method for real conditions functioning. First, the FV sends a "Start" command using visible light communication (VLC) [17], [18] to notify the LV that distance measurement is about to start. Once this command is received, the LV switches from an idle mode to the active mode, where the light signal collected by its
PD is reconstructed and used to drive its taillights. Therefore, the FV is able to perform the estimation of distance, using a first correction rule. After $Q$ consecutive measures, the FV sends a "Calibration" command to the LV using VLC and thus stops the range-finding process to start an auto-calibration step instead. This auto-calibration step consists in sending a square signal $s_{e}$ of frequency $f_{e}$ directly to the processing card and then measuring its phase-shift with the resulting reconstructed signal $s_{r}$. In the meantime, the LV performs the same operation and then sends the result using VLC to the FV so that the FV can mix the two processing delays measured to build a new correction rule that will replace the previous one. Finally, the FV can send a new "Start" command in order to enter the range-finding mode again.

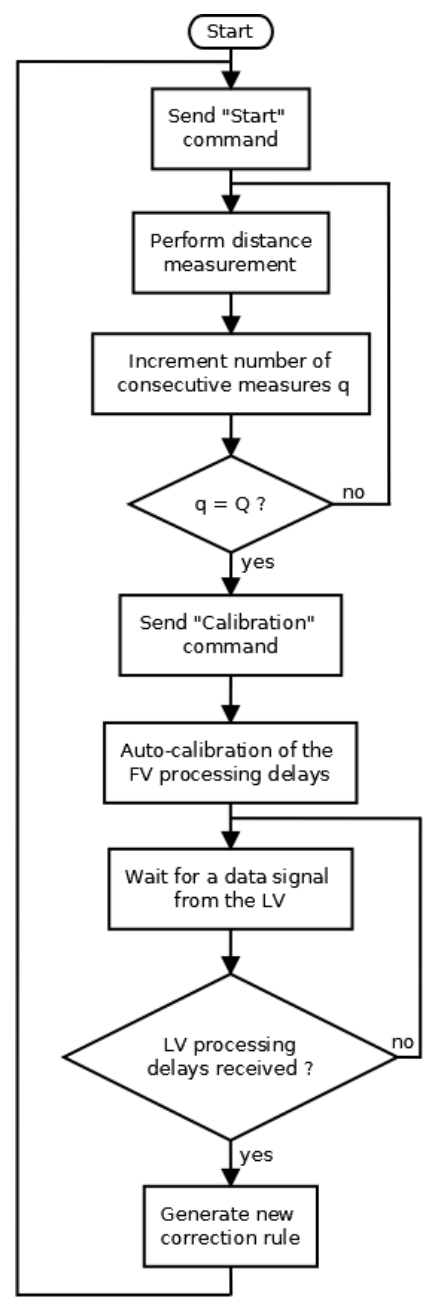

Fig. 11. Flow chart of the auto-calibration process of the VLR from the FV point of view.

\section{Measurement Error and Resolution}

The fixed correction rule previously exposed allows to remove the inflection point observed on Fig. 10 and thus a large part of the initial measurement error, defined as the difference between the distances output by the VLR and the true distances. However, there is still after correction a residual error. To characterize this error, we can study the distributions 
of the 4096 consecutive measures taken at each distance tested. Fig. 12(a) shows this distribution when the 'true' V2V distance is $18 \mathrm{~m}$ and before correction. It has a Gaussian-like shape with a specific standard deviation $\sigma$ of $17 \mathrm{~cm}$ and mean value around $7.8 \mathrm{~m}$. Note that the correction rule does not change this shape but simply shifts the mean value closer to zero. Consequently, we can introduce, in addition to the measurement error, the measurement resolution defined as twice the standard deviation, which gives for example a resolution of $37 \mathrm{~cm}$ at $18 \mathrm{~m}$. Then, when this operation is repeated for all the other V2V distances tested, similar distributions are obtained, even though the corresponding standard deviations $\sigma$, and thus the resulting measurement resolutions, increase with the distance, as highlighted by Table I.

(a)

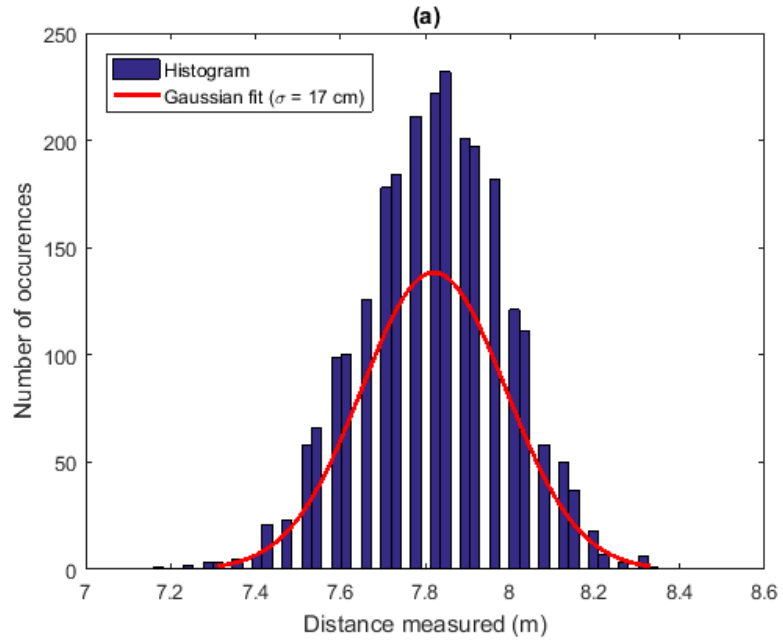

(b)

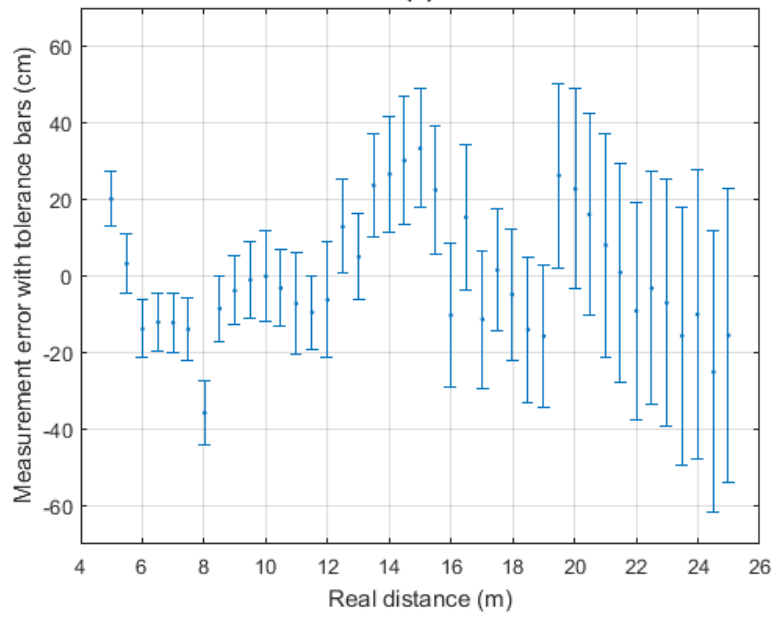

Fig. 12. (a) Distribution, before correction, of 4096 consecutive measurements performed at a 'true' $\mathrm{V} 2 \mathrm{~V}$ distance of $18 \mathrm{~m}$ (blue blocks) and resulting Gaussian fit (red curve), (b) evolution of the mean measurement error with the corresponding error bars after correction.

These variations can be explained by the phase noise of the signal reconstructed $s_{r}$, and thus by the limits of the hardware implementation of our VLR prototype. $s_{r}$ is indeed supposed to be a square signal of fixed frequency $f_{e}$ and duty cycle $50 \%$. However, the reconstruction process, mixed with the limited bandwidth of the emitters on both sides, cannot guarantee such a stability in frequency and duty cycle.
TABLE I

EVOLUTION OF THE DISTANCE RESOLUTION OF THE MEASUREMENT DISTRIBUTION WITH THE TRUE V2V DISTANCE.

\begin{tabular}{|c|c|}
\hline True distance (m) & $\begin{array}{c}\text { Measurement resolution } 2 \sigma \\
(\mathbf{c m})\end{array}$ \\
\hline 4.988 & 14.18 \\
\hline 7.483 & 16.44 \\
\hline 10.001 & 23.60 \\
\hline 12.49 & 24.52 \\
\hline 15.001 & 30.94 \\
\hline 17.498 & 31.68 \\
\hline 20.027 & 52.16 \\
\hline 22.499 & 60.90 \\
\hline 24.985 & 77.04 \\
\hline
\end{tabular}

The resulting fluctuations will necessarily vary the phase-shift between $s_{r}$ and the signal first sent $s_{e}$, and eventually the distance measured. Given the distribution represented on Fig. 12(a), we can deduce this phase noise is Gaussian and that it gets stronger with the V2V distance, so when the SNR of the signals received on both ends of the VLR decreases.

We can now plot on Fig. 12(b) the final evolution of the measurement error, with the blue dots denoting the mean errors, and error bars, separated by twice the standard deviation at the corresponding distance, meaning that $70 \%$ of the values output by the VLR will be contained in the interval they define. This figure also shows that our fixed correction rule removes efficiently the large initial errors observed on Fig. 11. The average errors are now indeed varying between $-35 \mathrm{~cm}$ and $35 \mathrm{~cm}$, without specific distribution, but with $75 \%$ of them actually between $-16 \mathrm{~cm}$ and $16 \mathrm{~cm}$. These results finally allow us to plot, on Fig. 13, the evolution of the distance measured by the VLR, after correction, and with the corresponding error bars. This curve demonstrates that our VLR is functional, even though the performance reached so far are still lower than the measurement resolution of $30 \mathrm{~cm}$ at $30 \mathrm{~m}$ found in the simulation analysis [9]. This difference in performance can mainly be explained by the phase noise of $s_{r}$ caused by the various distortions induced by the limited bandwidth of the transmit LED and of the PD, or by the filtering and comparison stages used to reconstruct $s_{r}$. This phase noise leads to phase-shift variations in $s_{\varphi}$ that, as shown by Fig. 4, can cause eventually large measurement errors. Therefore, our VLR implementation still needs to be improved.

\section{CONClusions And Future Works}

In this paper, a proof of concept of the VLR, already studied through simulations [9], [10], is brought for the first time. A prototype based on COTS automotive lights and PD combined with custom made LED drivers and analog signal processing cards is built and tested in order to demonstrate the rangefinding capabilities of our system. Although not optimized from the hardware point of view, this prototype enables distance measurement up to $25 \mathrm{~m}$ and ensures a measurement resolution of around $24 \mathrm{~cm}$ at $10 \mathrm{~m}$, with a refresh rate of $506 \mathrm{~Hz}$. As a reminder, highway platooning is optimal with a $\mathrm{V} 2 \mathrm{~V}$ distance of $8 \mathrm{~m}$, and the refresh rate of the measurement should reach at least $50 \mathrm{~Hz}$, with a few centimeters of errors. 


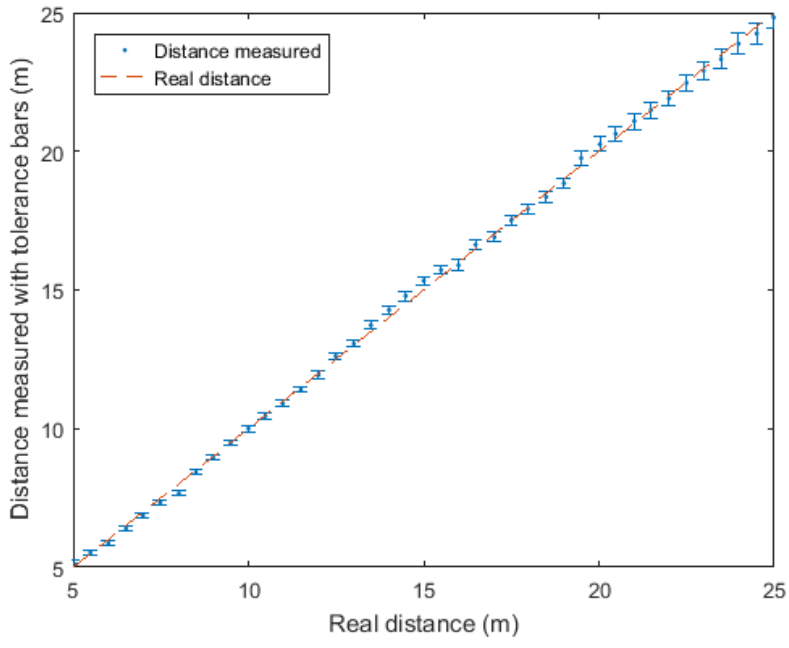

Fig. 13. Evolution of the distance measured after correction with the corresponding error bars (blue dots) and of the true V2V distance (red dashes).

These results thus show that the automotive lighting can be used for range-finding purposes, which would provide a simple and low cost redundant solution to existing lidar and radar. Although the current prototype use expensive off-the-shelf photo-receivers and FPGA development board, the rest of the components used account for less than $\$ 15$. Therefore, we can easily imagine that an optimized version of the VLR with dedicated components would be very cost-competitive. However, this optimization must be performed, starting with the improvement of the measurement resolution.

If the measurement errors can be smoothened by averaging several consecutive values $(N>1)$, the best way to enhance these performances it to mitigate the impact of the noise and thus improve the SNR of the signals received on both ends, which would limit the variations of the measures. In practice, since the optical power transmitted cannot be increased because of standard regulations, the only degrees of freedom are the light collection and reconstruction processes. From the light collection point of view, focusing lenses carefully designed could be used to optimize the amount of light detected by a front-end also improved in terms of gain and noise immunity. From the reconstruction step point of view, a better hardware design combined with a meticulous choice of the components would probably enhance the stability of the reconstructed signals. The resulting prototype would then be tested first under various lighting and interferences scenario and then in real traffic conditions to deepen the performance analysis. A data transmission, currently not supported by classical lidar or radar, could then be added by turning the square signal into a data signal and using clock recovery techniques to keep performing the phase-shift measure.

\section{REFERENCES}

[1] C. Bergenhem et al., "Overview of platooning systems," in Proc. 19th ITS World Congress, 2012.

[2] S. Tsugawa, S. Jeschke, and S. E. Shladover, "A Review of Truck Platooning Projects for Energy Savings," IEEE Trans. Intell. Veh., vol.1, no.1, pp. 68-77, Mar. 2016.
[3] P. S. Jootel. (2013, Jan.). SAfe Road TRains for the Environment Final Report. SARTRE Project. U.K. [Online] Available: http://www. sartre-project.eu/en/publications/Sidor/default.aspx.

[4] J. Hasch, "Driving towards 2020: Automotive Radar technology trends," in Proc. IEEE ICMIM, 2015, pp. 1-4.

[5] J. Lee, Y.-A. Li, M.-H. Hung, and S.-J. Huang, "A Fully-Integrated 77GHz FMCW Radar Transceiver in 65-nm CMOS Technology," IEEE J. Solid-State Circuits, vol. 45, no. 12, pp. 2746-2756, Dec. 2010.

[6] C. Niclass et al., "A 0.18-um CMOS SoC for a 100-m-Range 10-Frame/s 200 96-Pixel Time-of-Flight Depth Sensor," IEEE J. Solid-State Circuits, vol. 49, no. 1, pp. 315-330, Jan. 2014.

[7] M. Goppelt, H.-L. Blcher, and W. Menzel, "Automotive radar - investigation of mutual interference mechanisms," Adv. Radio Sci., vol. 8, pp. 55-60, Sep. 2010.

[8] G. Kim, J. Eom, and Y. Park, "An Experiment of Mutual Interference between Automotive LIDAR Scanners," in Proc. 12th ITNG, 2015, pp. 680-685.

[9] B. Béchadergue, L. Chassagne, and H. Guan, "Visible light phase-shift rangefinder for platooning applications," in Proc. 19th IEEE ITSC, 2016, pp. 2462-2468.

[10] B. Béchadergue, L. Chassagne, and H. Guan, "A visible light-based system for automotive relative positioning," accepted to IEEE Sensors, 2017.

[11] C.-Y. Wen, R. D. Morris, and W. A. Sethares, "Distance Estimation Using Bidirectional Communications Without Synchronous Clocking," IEEE Trans. Signal Process., vol. 55, no. 5, pp. 1927-1939, May 2007.

[12] S. Poujouly, B. Journet, and D. Miller, "Laser range finder based on fully digital phase-shift measurement," in Proc. 16th IEEE IMTC, 1999, pp. 1773-1776.

[13] S. Liu, J. Tan, and B. Hou, "Multicycle synchronous digital phase measurement used to further improve phase-shift laser range finding," Meas. Sci. Technol., vol. 18, no. 6, pp. 1756-1762, Jun. 2007.

[14] "Headlamps emitting an asymmetrical passing-beam," ECE Regulation No. 112 - Rev.3, Jan. 2013.

[15] "Position, stop and end-outline lamps," ECE Regulation No. 7 - Rev.6, Nov. 2012.

[16] B. Béchadergue, "Visible Light Range-Finding and Communication Using the Automotive LED Lighting," PhD Thesis, Université ParisSaclay (UVSQ), Nov. 2017.

[17] M. Y. Abualhoul, M. Marouf, O. Shagdar, and F. Nashashibi, "Platooning control using visible light communications: A feasibility study," in Proc. 16th IEEE ITSC, 2013, pp. 1535-1540.

[18] A.-M. Cailean et al., "Novel Receiver Sensor for Visible Light Communications in Automotive Applications," IEEE Sens. J., vol. 15, no. 8, pp. 4632-4639, Aug. 2015.

Bastien Béchadergue received the engineer degree from ISAE-Ensica, Toulouse, France and the M.S. in communication and signal processing from Imperial College London, London, UK in 2014. He also received in 2017 the Ph.D. in signal processing with the Vedecom Institute and the LISV laboratory at the University of Paris-Saclay, France. His current research interests include visible light communication and sensors.

Luc Chassagne received a B.S. in Electrical Engineering (1994) from Supelec (France) and received his Ph.D. (2000) in optoelectronics from the University of Paris XI, Orsay (France) for his work in the field of atomic frequency standard metrology. He is now Professor and Director of the LISV laboratory at the University of Versailles. The topics of interest in his research are nanometrology, sensors and Visible Light Communications.

Hongyu Guan received a B.S. in Electrical Engineering (2007) from ENSEIRB (France) and his Ph.D. (2012) in computer science from the University of Bordeaux I, Bordeaux (France) for his work on embedded systems for home automation. He is now Research associate and chief project at the LISV laboratory, University of Versailles. His current research interests are Visible Light Communications, ubiquitous, data fusion, sensors and nanometrology. 